\title{
ADAPTACIÓN TRANSCULTURAL DEL CUESTIONARIO "BARRIERS TO INCONTINENCE CARE SEEKING” EN MUJERES ADULTAS MAYORES EN EL ÁMBITO AMBULATORIO.
}

\section{CROSS-CULTURAL ADAPTATION OF THE "BARRIERS TO INCONTINENCE CARE SEEKING QUESTIONNAIRE” IN A ELDERLY WOMEN POPULATION IN AN OUTPATIENT CLINIC.}

\section{Viedma, Carolina Laura', Caruso, Diego².}

1 Médica Uróloga - Departamento de Urología, Unidad Asistencial Dr. César Milstein, Buenos Aires, Argentina. ORCID: http://orcid.org/0000-0001-9637-3722. Email de contacto: carogreen.87@hotmail.com

2 Médico. Magister en Investigación Clínica. Departamento de Investigación Clínica, Unidad Asistencial Dr. César Milstein, Buenos Aires, Argentina. ORCID: http://orcid.org/0000-0003-2981-7981. Email de contacto: diecaruso@hotmail.com

\section{Conceptos clave:}

Actualmente no existen trabajos en Argentina que informen acerca de las barreras que posee la población a la hora de consultar por incontinencia urinaria. En el siguiente artículo se realizó la adaptación transcultural del instrumento original BICS-Q y luego a través de entrevistas semi estructuradas se realizó una validación transcultural de su contenido. Creemos que este cuestionario puede abrir el debate para investigar estas barreras en otras regiones de nuestro país y buscar alternativas que faciliten la búsqueda de atención médica en mujeres con Incontinencia Urinaria.

\section{Resumen:}

Introducción: La incontinencia urinaria es una patología muy frecuente en la población femenina, sin embargo permanece oculto por la falta de percepción de la entidad como un problema. El objetivo de este estudio es adaptar transculturalmente al español el cuestionario "Barriers to Incontinence Care Seeking" (BISC-Q). Material y método: Se realizó la adaptación transcultural del Cuestionario (BICS-Q) en las siguientes etapas: traducción inicial, síntesis de las traducciones, retro-traducción, comité de expertos, pre-prueba y revisión del proceso de adaptación por los investigadores. Luego se realizó una validación de contenido a través de una encuesta buscando nuevas barreras que no formaban parte del cuestionario original. Las respuestas de las pacientes fueron unificadas y categorizadas dentro de los 5 dominios originales. Se incluyeron pacientes mujeres de más de 65 años en un centro ambulatorio especializado en adultos mayores. Resultados: Se incluyeron 164 mujeres con una edad media de 72 años (DE 6.4), de ellas $92(56 \%)$ refirieron pérdidas de orina en los últimos 12 meses, de las cuales 65 (71\%) nunca consultó al médico. Las barreras más prevalentes para consultar al médico estuvieron predominantemente relacionadas con: desconocimiento de la enfermedad, vergüenza e ideas equivocadas sobre el tratamiento. El nuevo cuestionario conserva tres de los cinco dominios del BICS-Q, se eliminó "costos" y "relacionado al sitio" y se incorporó un nuevo dominio sobre "falta de información". Conclusiones: El cuestionario BICS-Q fue adaptado para la evaluación de barreras para la consulta médica por incontinencia urinaria en mujeres mayores de 65 años de habla castellana.

Palabras clave: incontinencia urinaria; encuestas $y$ cuestionarios; comparación transcultural; anciano.

\begin{abstract}
:
Introduction: Urinary incontinence is a very frequent pathology in the female population, however it remains a hidden health problem. The main objective of the work is to cross-culturally adapt the BISC-Q questionnaire to be use spanish speaking population. Material and method: The cross-cultural adaptation of the Questionnaire (BICS-Q) was carried out in the following stages: initial translation, synthesis of the translations, retro-translation, expert committee, pretest and review of the adaptation process by the researchers. Then, a content validation was carried out through a survey looking for new barriers that were not part of the original questionnaire. The responses of the patients were unified and categorized within the 5 original domains. Women over 65 years of age were included in an outpatient specialized center for older adults. Results: The survey included 164 women, 56\% reported urine losses in the last 12 months, of which $71 \%$ never consulted the doctor about this problem. The answers about the barriers to consult were predominantly related to ignorance of the disease, shame and misconceptions about the treatment. The responses of the patients were unified and categorized within the 5 original domains. The new questionnaire retains three of the five domains of the BICS-Q, the domain "costs" and "related to the site" was removed and a new one was added on "lack of information". Conclusions: The Argentine version of the BICS-Q questionnaire is an instrument adapted for the evaluation of barriers to medical consultation due to urinary incontinence in spanish speaking women over 65 years of age.
\end{abstract}

Keywords: urinary incontinence; surveys and questionnaires; cross-cultural comparison; aged. 


\section{Introducción}

La Incontinencia Urinaria (IU) es un problema común en la población general en todo el mundo. En el estudio EPICONT 2, se evaluaron a más de 27.000 mujeres de un municipio de Noruega entre los años 2006 y 2008, la prevalencia encontrada de IU fue del $30 \%$ en mayores de 60 años ${ }^{1}$. Durante la 5 ta. Consulta Internacional sobre incontinencia en París en 2012 la prevalencia de IU entre los estudios de población femenina fue entre el $6 \%$ al $69 \%$, aunque la mayoría de los estudios que consultamos mostraron una prevalencia de IU entre el $25 \%$ y el $45 \%{ }^{2-5}$. Es probable que las tasas de prevalencia varíen debido a la falta de notificación de los síntomas de los pacientes, diferencias en los diseños de los estudios, el infra diagnóstico por parte de los médicos y diferencias culturales ${ }^{6-7}$. La IU afecta la capacidad de las mujeres para participar de actividades recreativas, laborales y sociales. Todo lo antedicho tiene efectos psicológicos negativos, como depresión, ansiedad y frustración $\mathrm{n}^{8-12}$.

A pesar de sus consecuencias, la IU permanece con frecuencia como un problema de salud oculto; sólo consultan entre el $20-50 \%$ de las pacientes, habitualmente con gran retraso ${ }^{13}$.

En un estudio en Estados Unidos Willis-Gray y col. encontraron que las latinas tienen más barreras para consultar por incontinencia que las mujeres de etnias blancas o negras ${ }^{14}$. En un estudio polaco realizado a 141 mujeres afirman que la ansiedad, la vergüenza y la convicción de que los síntomas de la IU son un estado normal relacionado con la edad, afectó significativamente el retraso en la búsqueda de consulta médica. La mayoría $(89,4 \%)$ de las mujeres encuestadas aliviaron los síntomas de la incontinencia urinaria sin la ayuda de un médico, a veces utilizando métodos perjudiciales para su salud tales como disminuir la actividad física y la cantidad diaria ingerida de líquido ${ }^{15}$. En oriente medio las barreras asociadas a la consulta por IU son diferentes y se relacionan principalmente con el vínculo entre la paciente y su esposo; así como también con el grado de interferencia que tenga la IU en la vida espiritual de la paciente por la posición adoptada en la plegarias ${ }^{16}$.

No hay un instrumento validado para evaluar las barreras en idioma español y en la cultura Argentina.

Nuestro objetivo principal es adaptar transculturalmente el cuestionario Barriers to Incontinence Care Seeking (BICS-Q) en una población de pacientes mujeres argentinas adultas mayores.

\section{Material y Método}

En 2008 se lleva a cabo el desarrollo de un cuestionario validado en múltiples etnias "Barriers to Incontinence Care Seeking" (BICS-Q) ${ }^{17}$; el cual tiene como objetivo principal conocer las barreras para la consulta médica por IU basándose en "The Theory of Care Seeking Behavior"18.

Se obtuvo la autorización del primer autor del cuestionario BICS-Q por correo electrónico.

El cuestionario original consta de 14 frases agrupados en 5 dominios según los distintos factores causales que limitan la consulta médica por IU.

El dominio de Relación (Relationship) evalúa la relación médico-paciente. El dominio de Costo (Cost) evalúa el costo que le genera una consulta médica al paciente. El dominio Inconvenientes con el sistema de salud (Inconvenience) se refiere a los problemas en obtención de turnos y a los tiempos en consultorio. El dominio Relacionado al sitio (Site-related) abarca la problemática de poder trasladarse a la clínica 0 consultorio. Por último, el dominio Miedo (Fear) evalúa los miedos del paciente a la hora de consultar.

En cuanto a la puntuación, se basó en la escala psicométrica tipo Likert. El paciente tiene 4 opciones de respuesta para cada frase según su grado de acuerdo con ella: "No estoy de acuerdo": 0 puntos. "Ligeramente de acuerdo": 1 punto. "Moderadamente de acuerdo": 2 puntos. "Totalmente de acuerdo": 3 puntos. A mayor puntaje total, mayor número de barreras y menor probabilidad de que ese paciente consulte al médico por IU.

\section{Traducción y adaptación transcultural}

Para iniciar la adaptación transcultural del instrumento se siguieron determinados pasos estructurados según los protocolos internacionales de transculturación y guías locales: traducción, traducción reversa, revisión por un comité, pretest y traducción final ${ }^{19-22}$. 
Para poder llevar a cabo la adaptación transcultural en nuestro medio y evaluar cuáles eran las barreras más frecuentes en nuestra población, se ideó un breve cuestionario que luego se comparó con las barreras presentes en el cuestionario original.

El estudio fue aprobado por el comité científico institucional y los pacientes dieron su consentimiento oral a la participación en el cuestionario.

Se realizó un estudio transversal entre el 1 de agosto y el 30 de septiembre de 2017. Se incluyeron pacientes de más de 65 años que aguardaban ser atendidas por consultas programadas en la sala de espera de múltiples especialidades en el centro ambulatorio de la Unidad Asistencial Dr. César Milstein. Este centro asistencial pertenece al Instituto Nacional de Servicios Sociales para Jubilados y Pensionados (INSSJP) y brinda atención médica a 30.000 pacientes por mes.

Fueron excluidas las pacientes que presentaban barreras del lenguaje (idiomáticas, ceguera, sordera o imposibilidad de comprender) y las que tenían consultas programados con las especialidades de urología y ginecología.

Las preguntas de la entrevista se encuentran en la Figura 1. La entrevista fue realizada siempre por la misma investigadora (mujer) para evitar el sesgo de deseabilidad social y el cuestionario fue completado en forma dirigida.

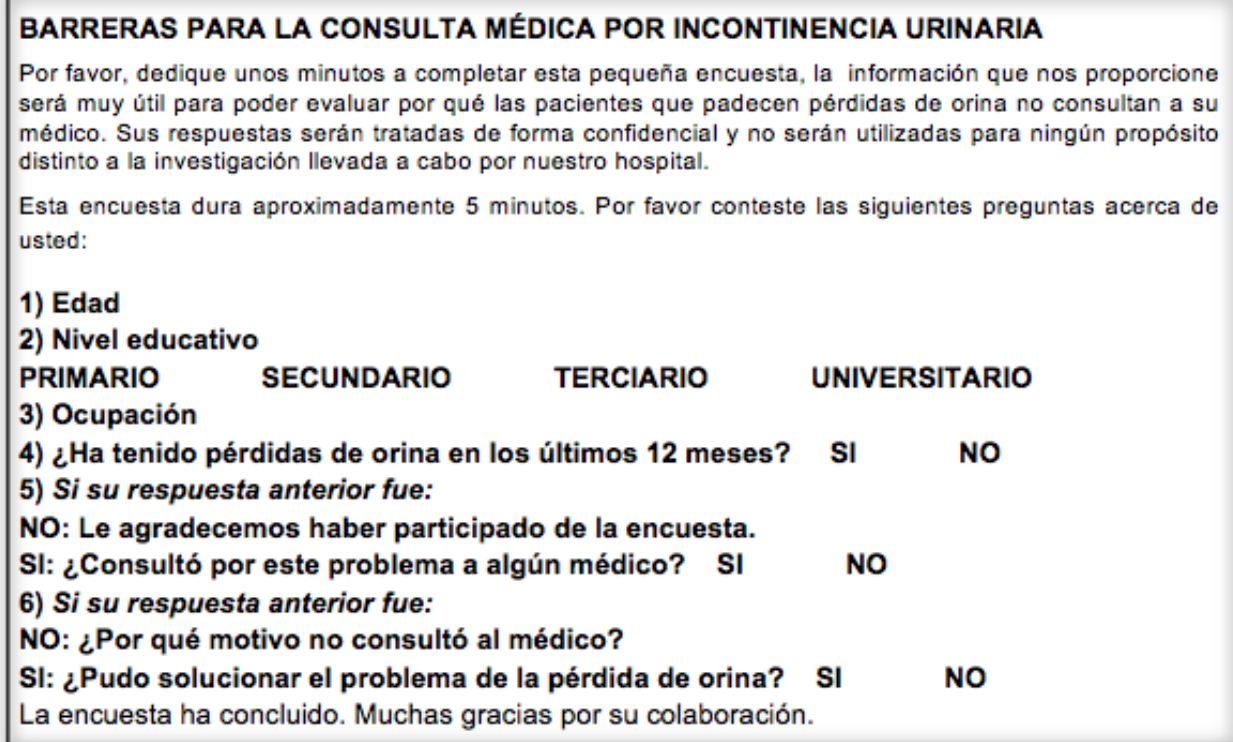

Figura $\mathrm{N}^{\circ}$ 1. Cuestionario ideado para recabar datos acerca de las barreras para la consulta por incontinencia urinaria en nuestra población.

Se recabaron datos demográficos como edad, nivel educativo y ocupación. Luego se les preguntó a las pacientes si habían tenido pérdidas de orina en los últimos 12 meses. A quien respondía afirmativamente se le preguntaba si había consultado al médico por su enfermedad y en caso de respuesta positiva, se le consultaba si había podido solucionar su problema. Si la respuesta era negativa se le preguntaba por qué no había consultado al médico.

Las respuestas abiertas a "por qué no consultó" nos permitieron conocer la problemática sin limitación. Luego de ser analizadas y agrupadas, estas afirmaciones fueron comparadas con el instrumento original y las diferencias incorporadas al cuestionario adaptado. Los dominios que no aparecieron por diferencias culturales, económicas, sociales y políticas con el país donde el cuestionario fue desarrollado, fueron eliminados de la versión adaptada.

\section{Análisis estadístico}

Las variables categóricas se reportan como frecuencia y proporciones con su IC95\%. Las variables continuas como media y desvío estándar.

El tamaño muestral se estimó para una frecuencia anticipada de incontinencia urinaria en mujeres adultas mayores de 30\%. Teniendo en cuenta una precisión absoluta de $+/-7.5$ con un Intervalo de confianza de $95 \%$ el tamaño de la muestra fue de 144 pacientes. El tipo de muestreo fue por conveniencia. 


\section{Resultados}

\section{Traducción}

Se realizó la traducción al idioma castellano. Para ello, se entregó la versión en inglés a dos traductores intérpretes bilingües nativos del idioma original; ambos profesionales contaban con reconocida experiencia en trabajos de traducción en ciencias de la salud. Terminada la traducción, se realizó una revisión del contenido del instrumento por expertos combinando ambas traducciones. Posteriormente se realizó una retro-traducción del castellano al idioma original. El instrumento original y la versión traducida fueron comparados para resolver las discrepancias entre los dos lenguajes. Ver Figura 2.

\section{"Barriers to Incontinence Care Seeking" (BICS-Q) en ingles original}

In the following section, I will ask about barriers to seeking care. To what extent do the following prevent you from seeking care for uncontrollable urine leakage. Please respond by answering not at all, slightly, moderately, or greatly. INTERVIEWER NOTE: READ AS OFTEN AS NECESSARY: HOW MUCH OF A BARRIER TO SEEKING CARE IS:

"I don 't have a health care practitioner who will see me for uncontrollable urine leakage". Relationship.

"There are long delays before insurance repay out-of-pocket expenses". Cost.

"My insurance too complicated to figure out". Cost.

"The cost of having my uncontrollable urine leakage evaluated is too high". Cost.

"There is no transportation to the office or clinic". Site-related.

"The wait is too long at the time of the appointment". Inconvenience.

"The office or clinic is too far away". Site-related.

"Appointments have to be scheduled too far ahead". Inconvenience.

"Office hours at the office or clinic are limited". Inconvenience.

"I don 't like to be examined or asked a lot of questions". Fear.

"The physician or nurse practitioner doesn't take time to explain what he or she is doing or why,

or. answer my questions". Relationship.

"I am afraid to find out if I have a serious problem". Fear.

"For some reason, I am afraid of the health care practitioner". Fear.

"The health care practitioner and his staff are not interested in my worries about my health". Relationship.

\section{BICS-Q traducido por expertos al idioma castellano}

En los siguientes enunciados hablaremos sobre las barreras del paciente a la hora de consultar con

el médico por incontinencia urinaria. Por favor, responda con una cruz en el recuadro que represente mejor su

sentimiento para cada frase. Leer la cantidad de veces que sea necesario:

¿Cuáles de estas barreras se le presentan ante la consulta medica por incontinencia urinaria?

"No tengo personal de salud a quien consultar por mi problema de pérdida de orina." Relación.

"Existen largas demoras por reintegros de la empresa de seguros por gastos particulares." Costo.

"Mi cobertura médica es demasiado compleja para entender." Costo.

"Es demasiado alto el costo de la consulta por incontinencia urinaria." Costo.

"No hay transporte al consultorio o a la clinica." Relacionado al sitio.

"La espera es demasiado larga a la hora de pedir el turno." Inconvenientes.

"El consultorio o la clínica están demasiado lejos." Relacionado al sitio.

"Hay que sacar el turno con demasiada anticipación." Inconvenientes.

"La atención del consultorio o clínica es demasiado corto." Inconvenientes.

"No me gusta cuando me examinan ni me preguntan demasiadas cosas." Miedo.

"El médico o la enfermera especialista no se toma el tiempo para explicarme lo que están haciendo

ni contestan mis preguntas." Relación.

"Me da miedo enterarme que tengo un problema serio." Miedo.

"Por alguna razón, el personal de salud me da miedo." Miedo.

"El médico y su equipo no están interesados en las preocupaciones que tengo sobre mi salud." Relación.

Figura $\mathrm{N}^{\circ}$ 2. Traducción del cuestionario original en idioma inglés al idioma castellano.

\section{Adaptación transcultural}

Fueron invitadas a participar un total de 185 mujeres, 164 aceptaron completar el cuestionario para la adaptación. El resto de las mujeres refirieron no tener tiempo para responder o se negaron por cuestiones personales. La media de edad de las mujeres encuestadas fue de 72 años. El $43 \%$ tenía entre 65 y 70 años. Con respecto al nivel educativo, el $45 \%$ (IC95\% 38-53) contaba con nivel educativo primario, un $40 \%$ (IC95\% 33-48) con nivel educativo secundario y un $15 \%$ (IC95\% 10-21) con nivel terciario/universitario. Respecto a la ocupación, el $91 \%$ afirmó ser jubilada o pensionada; el $9 \%$ restante era dueña de un comercio o empleada administrativa. 
Prevalencia de IU y barreras para la consulta médica

Del total de las 164 mujeres, 92 (56\% IC95 48-63) tenía o había tenido pérdidas de orina en los últimos 12 meses. De estas 92 mujeres, tan solo 27 (29\% IC95\% 21-39) había consultado alguna vez al médico por su problema. El 71\% restante dijo nunca haber consultado al médico, aunque padecía la enfermedad.

De las pacientes que consultaron al médico, sólo 9 mujeres (33\% IC95\% 18-52) pudieron solucionar el problema de incontinencia. Las 18 mujeres (67\%) restantes no obtuvieron buenos resultados después del tratamiento y persistieron con pérdidas de orina.

De las 65 mujeres con IU que no consultaron al médico, solo respondieron la pregunta "por qué no consultó" un $81 \%$. El $19 \%$ restante dijo no saber cuáles fueron las causas de la falta de consulta. Se utilizaron 53 respuestas válidas sobre las barreras que tenían para solicitar atención médica. Las respuestas se analizaron y agruparon en las frases que formaron parte del cuestionario final. Ver Tabla 1.

Tabla $\mathbf{N}^{\circ}$ 1: Barreras para la consulta por IU que surgieron a partir del cuestionario realizado en nuestra institución.

\begin{tabular}{l|c}
\multicolumn{1}{c|}{ Barreras para la consulta por incontinencia } & $\begin{array}{c}\text { Total mujeres: 53 } \\
(\mathbf{1 0 0 \%})\end{array}$ \\
\hline "habitualmente utilizo apósitos y me arreglo bien con ellos" & $11(21 \%)$ \\
"me da vergüenza decir que pierdo orina" & $8(15 \%)$ \\
"es normal a mi edad" & $6(11 \%)$ \\
"no tiene solución" & $5(9.5 \%)$ \\
"no tengo tiempo para concurrir al médico" & $5(9.5 \%)$ \\
"no sé cómo ni dónde sacar los turnos" & $4(7.5 \%)$ \\
"el médico no me explica lo que hace ni contesta mis preguntas" & $3(5.5 \%)$ \\
"me da miedo saber que tengo una enfermedad" & $2(4 \%)$ \\
"no sé a cuál médico ir" & $2(4 \%)$ \\
otras (ya reportadas en el cuestionario original) & $7(13 \%)$
\end{tabular}

Estas razones expresadas por las pacientes se compararon con las del cuestionario original. Se modificaron los 5 dominios originales ya que algunas frases originales no fueron respondidas por ninguna paciente y otras no formaban parte del original. Se obtuvieron 14 frases divididas en 4 dominios.

El dominio Costo no fue una variable manifestada por nuestra población. Esto se explica ya que el sistema de salud argentino difiere enormemente con el de Estados Unidos, el cual tiene un sistema de salud privado (seguros médicos), sin "salud pública" como en la Argentina. Como ésta problemática no apareció dentro de las razones dadas por las pacientes, se eliminó el dominio Costos de nuestro cuestionario.

El dominio Relacionado al sitio no fue una variable manifestada por nuestra población posiblemente porque los pacientes de PAMI tienen sedes de atención predeterminadas cercanas a sus viviendas.

El dominio Relación tuvo coincidencia con las tres frases del original antes descripto. Se agregó una cuarta frase que fue respuesta de numerosas mujeres (17\%) y no formaba parte del original: "me da vergüenza decir que pierdo orina". Por lo tanto se obtuvieron 4 frases dentro de este dominio.

El dominio Miedo contaba con 3 frases las cuales coincidían con las respuestas de nuestra encuesta, por lo tanto no fue modificado.

El dominio Inconvenientes con el sistema de salud contaba con tres frases. Las frases sobre "larga espera para poder sacar turnos" y "tiempos de atención muy cortos" coincidieron con las respuestas de nuestra encuesta. La frase "no sé cómo ni dónde sacar los turnos" se sumó a nuestro cuestionario ya que para muchas mujeres fue una barrera para la consulta. Por lo tanto la subescala Inconvenientes con el sistema sanitario contó con tres frases en total.

Por último se introdujo el dominio Falta de información, manifestado por el $43 \%$ de nuestras pacientes. Quedó conformado por tres opciones: "habitualmente uso apósitos y me arreglo bien con ellos", "es normal a mi edad" y "no tiene solución".

Queda así conformado el nuevo cuestionario en castellano adaptado transculturalmente. Se utilizó la misma estructura y el mismo tipo de puntuación tipo Likert para denotar mayor o menor número de barreras. Ver Figura 3. 


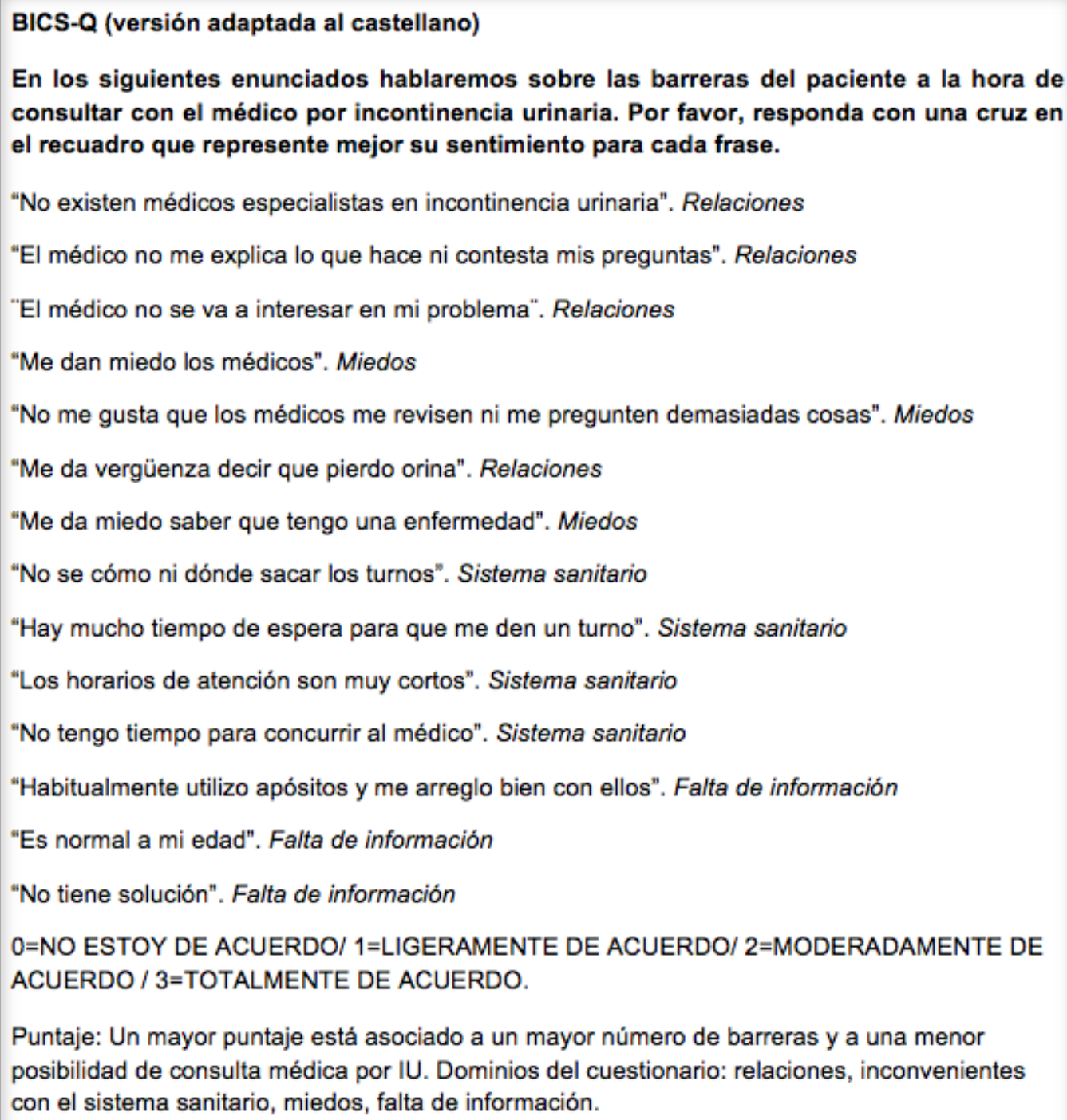

Puntaje: Un mayor puntaje está asociado a un mayor número de barreras y a una menor posibilidad de consulta médica por IU. Dominios del cuestionario: relaciones, inconvenientes con el sistema sanitario, miedos, falta de información.

Figura $\mathrm{N}^{\circ} 3$. Nuevo cuestionario en idioma castellano adaptado transculturalmente.

\section{Discusión}

De las 164 pacientes entrevistadas, un $56 \%$ manifestó haber tenido pérdidas de orina en el último año y solo un $29 \%$ de ellas solicitó atención médica por este motivo. Muchas de las razones que expresaron fueron coincidentes con las del diseño original pero el dominio de costos y el dominio relacionado con el sitio no aparecieron como barreras mientras que la falta de información fue un dominio nuevo.

Como fortaleza el presente estudio es el primero en nuestro país que aborda de manera específica el problema de la IU como un trastorno oculto y poco evaluado en el adulto mayor y permitiría dar el puntapié para comenzar a conocer las barreras a la consulta por IU en futuros estudios.

Una limitante de nuestro estudio es que fue adaptado en mujeres adultas mayores, ya que es un hospital que solo atiende pacientes adheridas a PAMI (siendo en su gran mayoría mayores de 65 años). No podemos entonces generalizarlo a toda la población femenina argentina.

Creemos que el dominio de "Costos" aparecería si hubiéramos realizado el cuestionario en otro escenario o nivel sociocultural, porque a pesar que la cobertura de salud es universal en nuestro país, los costos en transporte podrían ser un problema en la población con bajos recursos que requiere trasladarse para hacer la consulta.

Otra limitante del estudio es que realizamos la encuesta a mujeres dentro del hospital que ya están inmersas en el sistema de salud. Por lo tanto no abarca a la población que habitualmente no concurre al centro de salud. En este caso el dominio Relacionado el sitio podría ser una barrera presente en pacientes que viven lejos de consultorios u hospitales, donde hay falta de transporte.

Según una revisión realizada en una universidad chilena, los hallazgos revelan que la mayoría de los trabajos sobre las barreras y los facilitadores en la atención de la salud se han realizado con personas 
usuarias de los propios servicios de salud, por tanto la disponibilidad de los mismos no constituye una barrera frecuente. Es posible que quienes no contactan a los sistemas de atención sean grupos más vulnerables que quienes sí los contactan. Tal vulnerabilidad estaría asociada, entre otras razones, a la condición social, económica y cultural de estas poblaciones, motivando que las barreras que enfrentan sean más frecuentes y de mayor complejidad ${ }^{23}$.

A futuro, creemos importante validar el cuestionario en los demás rangos etarios, niveles socioeconómicos y grupos de personas que no contactan al sistema de salud. Una alternativa para incluir a estos grupos es desarrollar el cuestionario para ser utilizado en redes sociales o telefónicamente.

Si logramos llevar a cabo la encuesta a nivel nacional, los resultados nos permitirían realizar nuevas estrategias en la atención primaria y en la relación médico-paciente, para abordar los sentimientos de vergüenza y negación de nuestras pacientes. Así evitaríamos los retrasos en las consultas, los costes directos en el sistema sanitario e indirectos en los pacientes y mejoraríamos la calidad de vida de las mujeres que sufren IU.

Actualmente se está realizando la validación psicométrica del cuestionario para evaluar su estabilidad inter e intraobservador.

\section{Conclusión}

El cuestionario BICS-Q fue adaptado para la evaluación de barreras en la consulta médica por incontinencia urinaria en mujeres mayores de 65 años de habla castellana. Su uso está recomendado para la búsqueda de barreras para la consulta médica por incontinencia urinaria.

Limitaciones de responsabilidad: Ninguno.

Conflicto de intereses: Ninguno. La institución de la cual se recabaron los datos no es responsable de la publicación de esta investigación, ni de los resultados obtenidos de ella.

Fuentes de apoyo: la presente investigación no ha recibido ninguna beca especifica de agencias de los sectores público, comercial, o sin ánimo de lucro.

\section{Bibliografía}

1. Ebbesen, M. H., Hunskaar, S., Rortveit, G. \& Hannestad, Y. S. Prevalence, incidence and remission of urinary incontinence in women: longitudinal data from the Norwegian HUNT study (EPINCONT). BMC Urol. 13, 27 (2013).

2. Hunskaar, S., Lose, G., Sykes, D. \& Voss, S. The prevalence of urinary incontinence in women in four European countries. BJU Int. 93, 324-330 (2004).

3. Irwin, D. E. et al. Population-based survey of urinary incontinence, overactive bladder, and other lower urinary tract symptoms in five countries: results of the EPIC study. Eur. Urol. 50, 1306-14; discussion 1314-5 (2006).

4. Niang, L. et al. [Urinary incontinence in woman: epidemiologic profile in Sub Saharian countries]. Prog. Urol. 20 , 1213-1216 (2010).

5. Coyne, K. S., Margolis, M. K., Kopp, Z. S. \& Kaplan, S. A. Racial Differences in the Prevalence of Overactive Bladder in the United States From the Epidemiology of LUTS (EpiLUTS) Study. Urology 79, 95-101 (2012).

6. Searcy, J. A. R. Geriatric Urinary Incontinence. Nurs. Clin. North Am. 52, 447-455 (2017).

7. Qaseem, A. et al. Nonsurgical management of urinary incontinence in women: a clinical practice guideline from the American College of Physicians. Ann. Intern. Med. 161, 429-440 (2014).

8. Koch, L. H. Help-seeking behaviors of women with urinary incontinence: an integrative literature review. J. Midwifery Womens. Health 51, e39-44 (2006).

9. Felde, G., Ebbesen, M. H. \& Hunskaar, S. Anxiety and depression associated with urinary incontinence. A 10year follow-up study from the Norwegian HUNT study (EPINCONT). Neurourol. Urodyn. 36, 322-328 (2017).

10. White, A. J., Reeve, B. B., Chen, R. C., Stover, A. M. \& Irwin, D. E. Coexistence of urinary incontinence and major depressive disorder with health-related quality of life in older Americans with and without cancer. J. Cancer Surviv. 8, 497-507 (2014).

11. Vo, K., Forder, P. M. \& Byles, J. E. Urinary Incontinence and Social Function in Older Australian Women. J. Am. Geriatr. Soc. 64, 1646-1650 (2016).

12. Bedretdinova, D., Fritel, X., Zins, M. \& Ringa, V. The Effect of Urinary Incontinence on Health-related Quality of Life: Is It Similar in Men and Women? Urology 91, 83-89 (2016). 
13. Horrocks, S., Somerset, M., Stoddart, H. \& Peters, T. J. What prevents older people from seeking treatment for urinary incontinence? A qualitative exploration of barriers to the use of community continence services. Fam. Pract. 21, 689-696 (2004).

14. Willis-Gray, M. G., Sandoval, J. S., Maynor, J., Bosworth, H. B. \& Siddiqui, N. Y. Barriers to Urinary Incontinence Care Seeking in White, Black, and Latina Women. Female Pelvic Med. Reconstr. Surg. 21, 83-86 (2015).

15. Wójtowicz, U., Płaszewska-Zywko, L., Stangel-Wójcikiewicz, K. \& Basta, A. Barriers in entering treatment among women with urinary incontinence. Ginekol. Pol. 85, 342-347 (2014).

16. El-Azab, A. S. \& Shaaban, O. M. Measuring the barriers against seeking consultation for urinary incontinence among Middle Eastern women. BMC Womens. Health 10, 3 (2010).

17. Heit, M., Blackwell, L. \& Kelly, S. Measuring barriers to incontinence care seeking. Neurourol. Urodyn. 27, 174178 (2008).

18. Heit, M., Blackwell, L. \& Kelly, S. Adapting the Theory of Care Seeking Behavior to the Clinical Problem of Urinary Incontinence. J. Pelvic Med. Surg. 14, 29-35 (2008).

19. Beaton, D. E., Bombardier, C., Guillemin, F. \& Ferraz, M. B. Guidelines for the Process of Cross-Cultural Adaptation of Self-Report Measures. Spine 25, 3186-3191 (2000).

20. Guillemin, F., Bombardier, C. \& Beaton, D. Cross-cultural adaptation of health-related quality of life measures: Literature review and proposed guidelines. J. Clin. Epidemiol. 46, 1417-1432 (1993).

21. Escobar Bravo, M. Á. \& Bravo, M. Á. E. Adaptación transcultural de instrumentos de medida relacionados con la salud. Enfermería Clínica 14, 102-106 (2004).

22. Cardoso Ribeiro, C., Gómez-Conesa, A. \& Montesinos, M. D. H. Metodología para la adaptación de instrumentos de evaluación. Fisioterapia 32, 264-270 (2010).

23. Hirmas Adauy, M. et al. Barreras y facilitadores de acceso a la atención de salud: una revisión sistemática cualitativa. Rev. Panam. Salud Pública 33, 223-229 (2013). 